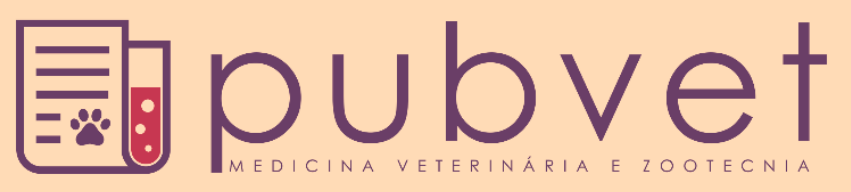

https://doi.org/10.31533/pubvet.v12n12a240.1-8

\title{
Perfil clínico e laboratorial da intoxicação canina por Ricinus communis: Relato de dois casos
}

\author{
Fabio Navarro Baltazar ${ }^{*} \bullet$, Mariana Haddad Capellanes ${ }^{1}$, Kimberly K. R. da Costa ${ }^{2} \bullet$, \\ Carla Alice Berl ${ }^{3}$ \\ ${ }^{1}$ Médicos veterinários do Departamento de Clínica Médica do Hospital Veterinário Pet Care, São Paulo, SP, Brasil. \\ ${ }^{2}$ Graduanda do curso de Medicina Veterinária das Faculdades Metropolitanas Unidas, UniFMU, São Paulo, SP, Brasil. \\ ${ }^{3}$ Médica veterinária, Diretoria Clínica, Hospital Veterinário Pet Care, São Paulo, SP, Brasil. \\ *Autor para correspondência, E-mail: fabionavarro@petcare.com.br
}

\begin{abstract}
RESUMO. As intoxicações por Ricinus communis encontram-se amplamente descritas em medicina humana e veterinária, ocasionando manifestações clínicas de intensidade distinta entre as espécies, na dependência da suscetibilidade individual, da quantidade ingerida e das partes ou subprodutos relacionados à planta ingeridos, como o adubo denominado torta de mamona. Os sinais e sintomas comumente observados nos pacientes intoxicados incluem principalmente manifestações gastrointestinais, porém há possibilidade de envolvimento do sistema circulatório nos quadros mais graves. O presente relato descreve dois animais da espécie canina (aqui denominados "paciente 1", da raça golden retriever, e "paciente 2", sem raça definida), admitidos no ambulatório do Hospital Veterinário Pet Care apresentando êmese e prostração após terem ingerido torta de mamona, durante a adubação do jardim domiciliar dos tutores. Ambos os animais permaneceram internados para terapia suporte sintomáticos intravenosos e foram submetidos à hematologia, bioquímica sérica completa, ultrassonografia abdominal, dosagem de lipase específica pancreática (SPEC), além de tempo de protrombina e de tromboplastina parcial ativada apenas para o cão que apresentou sinais sugestivos de pancreatite no referido exame imaginológico ("paciente 2"). As análises hematológicas revelaram apenas leucocitose, e os exames bioquímicos não apresentaram alterações, assim como as dosagens de lipase específica pancreática, porém observou-se aumento no tempo de tromboplastina parcial ativada. O período de hospitalização compreendeu 24 e 72 horas, respectivamente, uma vez que o animal sem raça definida ("paciente 2") apresentou-se hipotenso na admissão hospitalar e no período intensivo inicial. Ambos apresentaram evolução clínica favorável, tendo recebido alta no quarto dia após a ingestão do adubo. Desta maneira, conclui-se o prognóstico favorável na dependência da instituição terapêutica precoce, especialmente quando realizada de maneira intensiva.
\end{abstract}

Palavras chave: ricina, cães, intoxicação por plantas.

\section{Clinical and laboratorial profile of canine intoxication by Ricinus communis: Report of two cases}

ABSTRACT. Intoxications by Ricinus communis are widely described in human and veterinary medicine, with clinical manifestations being distinct in intensity among species, depending on the individual susceptibility, the amount ingested, and the parts or products of the plant ingested, such as the fertilizer castor bean. Signs and symptoms commonly observed in intoxicated patients include mainly gastrointestinal manifestations, although the involvement of circulatory system is possible, as seen in most severe cases. The present report describes two dogs (referred as "patient 1", golden retriever, and patient 2, a mongrel 
dog), admitted at the Pet Care Veterinary Hospital ambulatory presenting emesis and prostration after having eaten castor bean cake during the fertilization of the tutors' garden, at home. Both animals were hospitalized for symptomatic intravenous support therapy and underwent hematology, complete serum biochemistry, abdominal ultrasonography and pancreatic specific lipase (SPEC), although prothrombin time and activated partial thromboplastin time were performed only for the patient 2 , since pancreatitis was suspected. Hematological analysis only revealed leukocytosis, and biochemical tests showed no alterations, as well as pancreatic specific lipase dosages, although the increased activated partial thromboplastin time. The hospitalization period was 24 and 72 hours, respectively, since patient 2 was hypotensive at admission and initial intensive period. Both animals presented favorable clinical evolution until the fourth day of treatment, after the ingestion of castor bean cake. Therefore, we concluded the favorable prognosis depending on early therapeutic institution, especially when performed intensively.

Keywords: ricin, dogs, plant poisoning

\section{Perfil clínico y laboratorial de la intoxicación canina por Ricinus communis: Reporte de dos casos}

RESUMEN. Las intoxicaciones por Ricinus communis están ampliamente descritas en medicina humana y veterinaria, ocasionando manifestaciones clínicas de intensidad distinta entre las especies, en la dependencia de la susceptibilidad individual, de la cantidad ingerida $\mathrm{y}$ de porciones o subproductos relacionados a la planta, como el abono denominado torta de ricino. Los signos y síntomas comúnmente observados en los pacientes intoxicados incluyen principalmente manifestaciones gastrointestinales, pero hay posibilidad de involucrar el sistema circulatorio en los cuadros más graves. El presente relato describe dos animales de la especie canina (aquí denominados paciente 1, de la raza golden retriever, y paciente 2 , sin raza definida), admitidos en el ambulatorio del Hospital Veterinario Pet Care presentando vómitos y postración después de haber ingerido torta de ricino, durante la fertilización del jardín del domicilio de los tutores. Los dos animales permanecieron internados para terapia de apoyo sintomática intravenosa y se sometieron a hematología, bioquímica de la sangre completa, ultrasonografía abdominal, dosificación de lipasa específica pancreática (SPEC), además de tiempo de protrombina y de tromboplastina parcial activada sólo para el perro que presentó signos sugestivos de pancreatitis en el referido examen imaginológico ("paciente 2"). Los análisis hematológicos revelaron sólo leucocitosis, y los exámenes bioquímicos no presentaron alteraciones, así como las dosificaciones de lipasa específica pancreática, pero se observó un aumento en el tiempo de tromboplastina parcial activada. El período de hospitalización fue de 24 horas y 72 horas, respectivamente, una vez que el animal sin raza definida ("paciente 2") se presentó hipotenso en la admisión em la clinica y en el período intensivo inicial. Ambos animales presentaron evolución clínica favorable, habiendo recibido alta al cuarto día después de la ingestión de la torta de ricino. De esta manera, se concluye el pronóstico favorable en la dependencia de la institución terapéutica precoz, especialmente cuando se realiza de manera intensiva.

Palabras clave: ricina, perros, intoxicación por plantas

\section{Introdução}

Popularmente conhecida como mamona, Ricinus communis caracteriza-se por uma planta ornamental de origem africana amplamente utilizada no ramo industrial e medicinal (Roels et al. 2010). A planta contém ricina, uma toxalbumina (glicoproteína da família das lectinas) encontrada sob maior concentração no endosperma das sementes de mamona, apesar da variação observada entre os diferentes genótipos (Roels et al. 2010). A molécula de ricina é constituída por duas cadeias glicoproteicas (A e B), uma com atividade enzimática e outra com sítio de ligação específico ao açúcar galactose (Fonseca \& Soto-Blanco 2014). A cadeia B liga-se à parede celular pelas proteínas que contém galactose, permitindo à entrada da subunidade A por endocitose no citosol celular (Fonseca \& SotoBlanco 2014). A cadeia A, por sua vez, pertence a 
uma classe de enzimas que inibe a síntese protéica por meio da depurinação de ácido nucléico ribossomal 28S, levando a célula à morte (Albretsen et al. 2000), no contexto das intoxicações por esta substância. Tais intoxicações podem acometer diversas espécies de animais, como bovinos, equinos, caprinos, ovinos, suínos, aves domésticas, cães, gatos, e seres humanos, havendo susceptibilidade distinta na dependência da espécie envolvida (Tokarnia \& Döbereiner 1997). A toxicose em cães decorre da ingestão de 0,03 a 0,04 miligramas de ricina por quilograma de peso, com a dose letal encontrando-se entre $1 \mathrm{e}$ 2 gramas de semente por quilograma de massa corporal (Botha \& Penrith 2009). Nos cães, os sinais clínicos mais comuns são apatia, êmese e enterite hemorrágica, apesar de poderem ocorrer, de maneira menos frequente, anorexia, hematêmese, dor abdominal, sialorréia, ataxia, fraqueza e taquicardia (Botha \& Penrith 2009).

O presente trabalho tem por objetivo descrever os principais aspectos clínicos, laboratoriais e imaginológicos relacionados a dois pacientes da espécie canina, contactantes, intoxicados por Ricinus communis por meio da ingestão do adubo de mamona.

\section{Relato do caso}

$\mathrm{O}$ atual relato envolve dois cães do mesmo tutor, um golden retriever com 3 anos e 2 meses de idade (aqui denominado "paciente um"), e seu contactante sem raça definida, com 1 ano de idade (denominado "paciente dois"). Ambos os pacientes ingeriram torta de mamona durante a adubação do jardim domiciliar do tutor, instantes antes da admissão hospitalar, tendo sido este o critério diagnóstico utilizado para elucidação das manifestações clínicas de ambos, os quais apresentavam, do ponto de vista sintomático, êmese e prostração. O primeiro dos animais acima descritos (paciente 1) apresentou, em seu exame físico, manutenção da coloração de mucosas, normotermia $\left(38,4^{\circ} \mathrm{C}\right)$, aproximadamente $8 \%$ de desidratação, normoglicemia $(85 \mathrm{mg} / \mathrm{dl})$, lactato sérico de $3,3 \mathrm{mmol} / \mathrm{dl}$, e $130 \mathrm{mmhg}$ de pressão arterial sistólica. Já seu contactante, sem raça definida (paciente dois), revelou mucosas normocoradas, hipertermia $\left(40^{\circ} \mathrm{C}\right)$, desidratação moderada (em torno de $10 \%$ ), normoglicemia (97 $\mathrm{mg} / \mathrm{dl}), 2 \mathrm{mmol} / \mathrm{dl}$ de lactato venoso e pressão arterial sistólica de $94 \mathrm{mmHg}$. Ambos os animais foram submetidos à ultrassonografia abdominal, a qual revelou, similarmente, espessamento gastrointestinal, principalmente em cólon (Figura 1). Adicionalmente, ainda no referido exame, o paciente um apresentou evidências de hipomotilidade gastrointestinal, esplenomegalia e linfoadenomegalia jejunal, e seu contactante sem raça definida, aumento das dimensões pancreáticas (Figura 2), hiperecogenicidade peritoneal adjacente, linfoadenomegalia cecal e cólica.

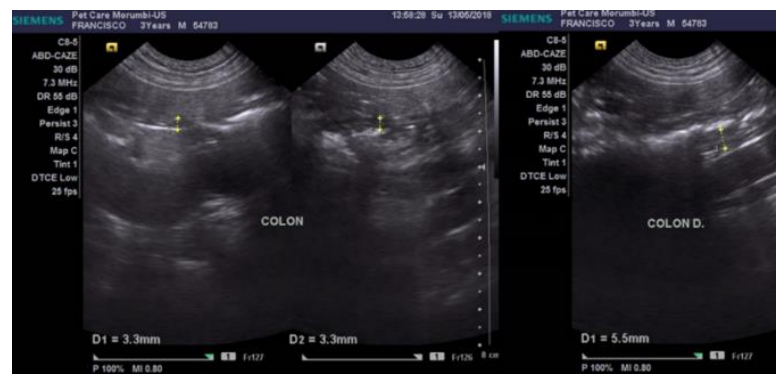

Figura 1. Exame ultrassonográfico abdominal do paciente 1 (golden retriever) revelando espessamento de parede do cólon transverso $(0,33 \mathrm{~cm})$ e de cólon descendente $(0,55 \mathrm{~cm})$ além de estratificação menos definida e alguns segmente discretamente corrugados (padrão inflamatório).

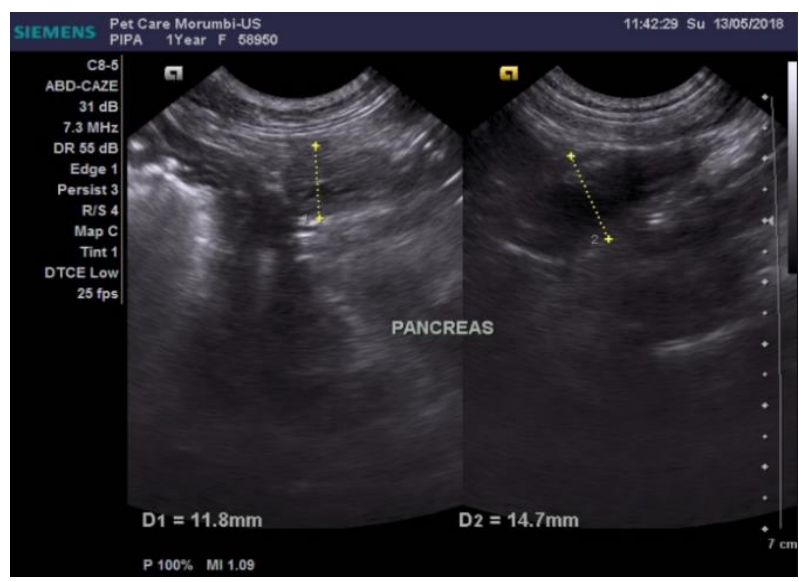

Figura 2: Exame ultrassonográfico abdominal do paciente 2 (sem raça definida) revelando pâncreas com dimensões aumentadas (medindo cerca de 1,2 centímetros de espessura no lobo esquerdo e 1,4 centímetros no lobo direito), além de ecogenicidade reduzida (aspectos sugestivos de pancreatite).

Os pacientes permaneceram internados para terapia sintomática parenteral e exames séricos complementares, incluindo hemograma, bioquímica completa, lipase específica pancreática, e tempos de protrombina e de tromboplastina parcial ativada apenas para $\mathrm{o}$ animal sem raça definida ("paciente 2"), devido à evidência ultrassonográfica de pancreatite. Os índices hematológicos dos cães sob debate podem ser visualizados nas tabelas 1 , assim como as análises bioquímicas, na tabela 2 . A dosagem de lipase específica pancreática revelou-se $58 \mu \mathrm{g} / \mathrm{L}$ para o paciente 1 , e $62 \mu \mathrm{g} / \mathrm{L}$ para o paciente 2 (referência 0 a $200 \mu \mathrm{g} / \mathrm{L}$ em cães). Já os tempos de coagulação foram de 13 segundos (referência 11 a 17 segundos) e 120 segundos (referência 72 a 102 segundos), respectivamente. 
Tabela 1. Hemograma dos pacientes da raça Golden Retriever (“paciente 1") e Sem raça definida ("paciente 2") intoxicado por Ricinuscommunis.

\begin{tabular}{|c|c|c|c|}
\hline & $\begin{array}{l}\text { Valor absoluto do } \\
\text { Paciente } 1\end{array}$ & $\begin{array}{l}\text { Valor absoluto do } \\
\text { paciente } 2\end{array}$ & $\begin{array}{c}\text { Valor relativo } \\
\text { Golden Retriever/Sem raça definida }\end{array}$ \\
\hline Índice de reticulócitos & - & - & 0,12 \\
\hline Leucócitos totais & $\begin{array}{c}26,06 \mathrm{mil} / \mathrm{mm}^{3}(6 \text { a } 17 \\
\left.\mathrm{mil} / \mathrm{mm}^{3}\right)\end{array}$ & $\begin{array}{c}24,41 \mathrm{mil} / \mathrm{mm} 3(8 \text { a } 16 \\
\mathrm{mil} / \mathrm{mm} 3)\end{array}$ & - \\
\hline Mielócitos & $0 / \mathrm{mm}^{3}$ & $0 / \mathrm{mm}^{3}$ & $0 \%$ \\
\hline Metamielócitos & $0 / \mathrm{mm}^{3}$ & $0 / \mathrm{mm}^{3}$ & $0 \%$ \\
\hline Bastonetes & $521,2 / \mathrm{mm}^{3}\left(0\right.$ a $\left.300 / \mathrm{mm}^{3}\right)$ & $244,1 / \mathrm{mm}^{3}\left(0\right.$ a $\left.300 / \mathrm{mm}^{3}\right)$ & $2 \%(0$ a $1 \%) / 1 \%(0$ a $1 \%)$ \\
\hline Segmentados & $\begin{array}{c}24.757 / \mathrm{mm}^{3}(3.600 \mathrm{a} \\
\left.13.000 / \mathrm{mm}^{3}\right)\end{array}$ & $\begin{array}{c}21.724,9 / \mathrm{mm}^{3}(4.400 \mathrm{a} \\
\left.11.200 / \mathrm{mm}^{3}\right)\end{array}$ & $95 \%$ (60 a $77 \%) / 89 \%$ (55 a 70\%) \\
\hline Eosinófilos & $0 / \mathrm{mm}^{3}\left(120\right.$ a $\left.1.700 / \mathrm{mm}^{3}\right)$ & $488,2 / \mathrm{mm}^{3}\left(80\right.$ a $\left.960 / \mathrm{mm}^{3}\right)$ & $0 \%(2$ a $10 \%) / 2 \%(1$ a $6 \%)$ \\
\hline Basófilos & $0 / \mathrm{mm}^{3}\left(0\right.$ a $\left.160 / \mathrm{mm}^{3}\right)$ & $0 / \mathrm{mm}^{3}\left(0\right.$ a $\left.160 / \mathrm{mm}^{3}\right)$ & $0 \%(0$ a $1 \%)$ \\
\hline Linfócitos & $521,2 / \mathrm{mm}^{3}\left(720\right.$ a $\left.5.100 / \mathrm{mm}^{3}\right)$ & $976,4 / \mathrm{mm}^{3}\left(1.600\right.$ a $\left.6.400 / \mathrm{mm}^{3}\right)$ & $2 \%(12$ a $30 \%) / 4 \%(20$ a $40 \%)$ \\
\hline Monócitos & $260,6 / \mathrm{mm}^{3}\left(60\right.$ a $\left.1.400 / \mathrm{mm}^{3}\right)$ & $976,4 / \mathrm{mm}^{3}\left(160\right.$ a $\left.1.200 / \mathrm{mm}^{3}\right)$ & $1 \%(3$ a $10 \%) / 4 \%(2$ a $8 \%)$ \\
\hline Plaquetas & $319 \times 10^{3} / \mathrm{mm}^{3}$ & $226 \times 10^{3} / \mathrm{mm}^{3}$ & 200 a $500 \times 10^{3} / \mathrm{mm}^{3}$ \\
\hline Proteína total & - & - & $7(5,5$ a 8 g/dl) /6,2 (5 a $7 \mathrm{~g} / \mathrm{dl})$ \\
\hline
\end{tabular}

Tabela 2. Exames bioquímicos dos pacientes da raça Golden Retriever (“paciente 1") e Sem raça definida (“paciente 2") intoxicado por Ricinuscommunis.

\begin{tabular}{|c|c|c|c|}
\hline Exames bioquímicos & $\begin{array}{l}\text { Resultado "paciente 1": } \\
\text { Golden Retriever }\end{array}$ & $\begin{array}{l}\text { Resultado "paciente } 2 \text { : } \\
\text { Sem raça definida }\end{array}$ & $\begin{array}{l}\text { Valores de } \\
\text { referência }\end{array}$ \\
\hline Bilirrubina total & $0,16 \mathrm{mg} / \mathrm{dl}$ & $0,16 \mathrm{mg} / \mathrm{dl}$ & 0,1 a $0,7 \mathrm{mg} / \mathrm{dl}$ \\
\hline Bilirrubina direta & $0 \mathrm{mg} / \mathrm{dl}$ & $0,11 \mathrm{mg} / \mathrm{dl}$ & 0,06 a $0,30 \mathrm{mg} / \mathrm{dl}$ \\
\hline Bilirrubina indireta & $0,16 \mathrm{mg} / \mathrm{dl}$ & $0,05 \mathrm{mg} / \mathrm{dl}$ & 0,01 a $0,50 \mathrm{mg} / \mathrm{dl}$ \\
\hline Cloretos & $109 \mathrm{mEq} / \mathrm{L}$ & $114 \mathrm{mEq} / \mathrm{L}$ & 108 a $128 \mathrm{mEq} / \mathrm{L}$ \\
\hline Cálcio total & $10,30 \mathrm{mg} / \mathrm{dl}$ & $9,90 \mathrm{mg} / \mathrm{dl}$ & 8,0 a $12,0 \mathrm{mg} / \mathrm{dl}$ \\
\hline Colesterol & $256 \mathrm{mg} / \mathrm{dl}$ & $229,20 \mathrm{mg} / \mathrm{dl}$ & 116 a $300 \mathrm{mg} / \mathrm{dl}$ \\
\hline Creatinina & $1,10 \mathrm{mg} / \mathrm{dl}$ & $0,70 \mathrm{mg} / \mathrm{dl}$ & 0,8 a $1,4 \mathrm{mg} / \mathrm{dl}$ \\
\hline Fosfatase alcalina & 90,50 U.I./L & 118,90 U.I./L & 10,0 a 96,0 U.I./L \\
\hline Gama-glutamil transferase & 4,40 U.I./L & 2,7 U.I./L & 1,2 a 6,4 U.I./L \\
\hline Glicose & $52,80 \mathrm{mg} / \mathrm{dl}$ & $86 \mathrm{mg} / \mathrm{dl}$ & 60,0 a $118,0 \mathrm{mg} / \mathrm{dl}$ \\
\hline Potássio & $4,30 \mathrm{mEq} / \mathrm{L}$ & $3,3 \mathrm{mEq} / \mathrm{L}$ & 4,1 a $5,4 \mathrm{mEq} / \mathrm{L}$ \\
\hline Albumina & $4,0 \mathrm{~g} / \mathrm{dl}$ & $3,7 \mathrm{~g} / \mathrm{dl}$ & 2,3 a $3,8 \mathrm{~g} / \mathrm{dl}$ \\
\hline Globulinas & $3,30 \mathrm{~g} / \mathrm{dl}$ & $2,5 \mathrm{~g} / \mathrm{dl}$ & 2,4 a $4,8 \mathrm{~g} / \mathrm{dl}$ \\
\hline Sódio & $152,0 \mathrm{mEq} / \mathrm{L}$ & $148 \mathrm{mEq} / \mathrm{L}$ & 143 a $168 \mathrm{mEq} / \mathrm{L}$ \\
\hline ALT (T.G.P.) & 48,20 U.I./L & 38,80 U.I./L & 7,0 e 92,0 U.I./L \\
\hline AST (T.G.O.) & 70,88 U.I./L & 65,40 U.I./L & 10 a 88 U.I./L \\
\hline Triglicérides & $48,10 \mathrm{mg} / \mathrm{dl}$ & $23,90 \mathrm{mg} / \mathrm{dl}$ & 15 a $150 \mathrm{mg} / \mathrm{dl}$ \\
\hline Uréia & $27,10 \mathrm{mg} / \mathrm{dl}$ & $16,6 \mathrm{mg} / \mathrm{dl}$ & 15,0 a $40,0 \mathrm{mg} / \mathrm{dl}$ \\
\hline Fósforo & $4,30 \mathrm{mg} / \mathrm{dl}$ & $4,80 \mathrm{mg} / \mathrm{dl}$ & 2,6 a $6,8 \mathrm{mg} / \mathrm{dl}$ \\
\hline
\end{tabular}


O cão da raça golden retriever (paciente um), o qual não apresentava evidências ultrassonográficas de pancreatite, permaneceu internado apenas por 24 horas, quando foi submetido ao seguinte manejo terapêutico: fluidoterapia intravenosa (solução de ringer com lactato, sob taxa de infusão de $5 \mathrm{ml} / \mathrm{kg} / \mathrm{H}$ ), metoclopramida $(0,3 \mathrm{mg} / \mathrm{kg} / \mathrm{H}$ I.V. a cada 12 horas), cloridrato de ranitidina $(2 \mathrm{mg} / \mathrm{kg}$ S.C. a cada 8 horas), omeprazol (1 mg/kg I.V. a cada 12 horas), citrato de maropitant ( $1 \mathrm{mg} / \mathrm{kg} \mathrm{S.C.} \mathrm{a} \mathrm{cada}$ 24 horas), metronidazol (15 mg/kg I.V. a cada 12 horas), carvão ativado ( $3 \mathrm{~g} / \mathrm{kg}$ V.O. a cada 8 horas), sucralfato $(50 \mathrm{mg} / \mathrm{kg}$ V.O. a cada 12 horas), cloridrato de ondasetrona $(0,5 \mathrm{mg} / \mathrm{kg}$ I.V. a cada 12 horas), e dipirona sódica ( $25 \mathrm{mg} / \mathrm{kg}$ I.V. a cada 8 horas). Foram realizadas três mensurações da pressão intra-abdominal nas 24 horas de terapia intensiva, com valores próximos de 5 centímetros de água (referência 0 a 5 centímetros de água). A evolução clínica foi favorável, com o animal não tendo apresentado novos episódios gastroentéricos em ambiente hospitalar, apesar de hiporexia persistente. Subsequentemente, a liberação foi realizada com prescrições de cloridrato de ranitidina (dose acima citada), domperidona $(0,1$ $\mathrm{mg} / \mathrm{kg}$ V.O. a cada 12 horas) e metronidazol (dose referida anteriormente). $\mathrm{O}$ paciente retornou ao hospital 2 dias e 5 dias após a liberação da terapia intensiva para reavaliação clínica, quando não mais apresentava distúrbios do apetite e alterações nos parâmetros avaliados, tendo recebido, na última destas datas, alta médica.

O paciente 2, por sua vez, permaneceu sob terapia intensiva por 72 horas, período que envolveu manejo terapêutico similar ao de seu contactante (anteriormente referido), com exceção de metoclopramida, e adição de enrofloxacina (5 $\mathrm{mg} / \mathrm{kg}$ S.C. a cada 12 horas). Neste período, não apresentou novos episódios eméticos, mas sim diarréias intermitentes, além de manutenção da leve hipotensão observada na admissão hospitalar nas primeiras 24 horas, com a pressão arterial sistólica tendo permanecido em torno de 96 $\mathrm{mmHg}$, mesmo sob fluidoterapia intravenosa ininterrupta, elevando-se para $112 \mathrm{mmHg}$ ao final das 72 horas intensivas. A pressão intra-abdominal revelou valores similares ao seu contactante. Alguns índices hematológicos e bioquímicos foram repetidos ao final desta hospitalização, como hematócrito $(42,8 \%)$, leucócitos $\left(14.910 / \mathrm{mm}^{3}\right)$, contagem de plaquetas $(255 \mathrm{x}$ $10^{3} / \mathrm{mm}^{3}$ ), tempo de protrombina (14 segundos) e de tromboplastina parcial ativada (133 segundos).
Ainda, neste mesmo momento, observou-se em controle ultrassonográfico abdominal ínfima coleção de efusão peritoneal e diminuição da ecogenicidade hepática, bem como a manutenção da descrição morfológica e topográfica das demais estruturas comparativamente ao exame anterior. Subsequentemente, com a normalização do apetite, o animal em questão foi liberado com prescrições de omeprazol, enrofloxacina, metronidazol e sucralfato, sob as mesmas doses já citadas. Após 24 horas, o paciente retornou ao hospital para reavaliação clínica, momento no qual houve relato de exacerbação do quadro diarréico e nova internação para manutenção dos procedimentos terapêuticos parenterais anteriormente iniciados, por 24 horas. Com a resolução do quadro entérico neste período, o animal foi liberado mediante a manutenção do tratamento já prescrito, e recebeu alta médica após 4 dias.

\section{Discussão}

As sementes de mamona, devido à sua impermeabilidade a água, podem não ocasionar quadros de intoxicação quando ingeridas por inteiro, ou seja, sua toxicidade se apresenta diretamente dependente do nível de maceração destas estruturas para subsequente liberação da toxalbumina (Roels et al. 2010). Por este motivo, em cães, as intoxicações decorrem, majoritariamente, da mastigação das sementes ou da ingestão de torta de mamona, um subproduto da cadeia produtiva da mamona, elaborado por meio da extração do óleo de suas sementes, com a finalidade de emprego como adubo orgânico (Soto-Blanco et al. 2002). Este adubo possui características superiores a de outros subprodutos orgânicos, a exemplo do esterco bovino, uma vez que fornece maiores concentrações de cálcio, potássio, fósforo e nitrogênio, desempenhando ainda efeito controlador de nematóides do solo e possuindo alto valor de proteínas (Cangemi et al. 2010). O emprego deste adubo em áreas com a presença de animais domésticos aumenta o risco de intoxicações, uma vez que a ricina, presente sob concentração de 3 a $5 \%$ na torta de mamona, se encontra livre para absorção (Hong et al. 2011). A toxicose em cães decorre da ingestão de 0,03 a 0,04 miligramas de ricina por quilograma de peso, com a dose letal encontrando-se entre 1 e 2 gramas de semente por quilograma de massa corporal (Botha \& Penrith 2009). Os sinais clínicos mais comuns, observados nos quadros de intoxicação canina, são apatia, êmese e enterite hemorrágica, apesar de poderem ocorrer, de maneira menos 
frequente, anorexia, hematêmese, dor abdominal, sialorréia, ataxia, fraqueza e taquicardia (Botha \& Penrith 2009)

O diagnóstico da intoxicação por rícino, por sua vez, pode ser realizado por meio da observação da ingestão de partes da planta (incluindo suas sementes e subprodutos) pelos animais, como procedido para os pacientes aqui relatados, porém outras modalidades são descritas em literatura, como pelo seu encontro no conteúdo emético ou nas fezes, por ensaio imunoenzimático, radioimunoensaio e cromatografia líquida associada à espectrofotometria de massas (Roels et al. 2010). Logo, devido à inespecificidade dos sinais e sintomas, tais análises bioquímicas podem ser úteis quando a informação de ingestão da planta não for relatada pelos tutores.

No contexto sintomático, ambos os animais deste estudo apresentavam êmese e prostração durante a admissão hospitalar, corroborando, desta maneira, a literatura relacionada ao tema no que se refere às manifestações gastrointestinais sobrepujarem outros eventuais sinais clínicos (Botha \& Penrith 2009). Quadros emético profusos associado à prostração apresentam-se, ainda, como os sintomas mais frequentes, porém o envolvimento do trato gastrointestinal, de maneira geral, pode ser explicado por meio da toxicidade celular do rícino. Após sua internalização pelas células, especialmente a nível gastrointestinal, ocorrerá a sequência de eventos bioquímicos intracelulares anteriormente citada $\mathrm{e}$ as manifestações clínicas descritas, incluindo abdominalgia e diarreia, a qual pode cursar com hematoquesia. O paciente dois, adicionalmente, apresentou hipertermia $\left(40^{\circ}\right.$ C) e hipotensão (pressão arterial sistólica de $94 \mathrm{mmhg}$ ), sinais já descritos em literatura, com tal elevação da temperatura corpórea tendo sido observada em aproximadamente $7 \%$ dos casos, segundo estudo realizado por Albretsen et al. (2000). Tais situações clínicas aventam fortemente a possibilidade de absorção do rícino para a via sistêmica, uma vez que estudos com a introdução desta substância por via parenteral já demonstraram biodistribuição principalmente para o fígado, baço e músculos, caracterizando danos hepáticos reversíveis, injúria endotelial vascular, edema e mialgias ( $\mathrm{Fu}$ et al. 1996). A deterioração da função cardiovascular, nestes casos, pode ainda ser potencializada por necrose miocárdica e hemorragia cardíaca, secundariamente ao distúrbio na homeostase do cálcio, uma vez que há o decréscimo na absorção deste eletrólito pelo retículo sarcoplasmático.

O estudo hematológico de ambos os pacientes revelou leve leucocitose por neutrofilia e concomitante desvio à esquerda, além de linfopenia, fatos que podem ser atribuídos ao processo inflamatório gastrointestinal presente no momento da admissão hospitalar, à eventual inflamação sistêmica (cardiovascular e musculoesquelética) ocorrida pela suspeita de absorção da ricina, e ainda à liberação maciça de cortisol ocorrida durante situações de depleção orgânica, configurando leucograma de estresse. As análises bioquímicas referentes ao paciente um (Golden Retriever) demonstraram apenas leve hipoglicemia, porém tal situação certamente não reflete a condição real da concentração de glicose sérica do paciente no momento do atendimento, visto que o tempo transcorrido entre a coleta de sangue e seu processamento em ambiente laboratorial pode predispor à queda na concentração desta molécula na amostra (Aleixo et al. 2010) proposta esta corroborada pela mensuração glicêmica durante a admissão hospitalar, como acima citado. Já o paciente dois (SRD) apresentou leve aumento na enzima fosfatase alcalina e moderada hipocalemia, alterações que reforçam a intensidade do processo inflamatório gastrointestinal.

Interessantemente, o paciente dois (SRD) apresentou sinais ultrassonográficos compatíveis com pancreatite, incluindo aumento das dimensões e hipoecogenicidade desta víscera, porém a dosagem de lipase pancreática por imunorreatividade (Spec CPL) não confirmou esta suspeita, tendo se apresentado dentro dos limites de normalidade. Baltazar et al. (2016) descreveram 71 casos de pancreatite aguda em cães, onde o critério diagnóstico para seleção destes pacientes foi exame ultrassonográfico abdominal compatível com tal afecção, ou ainda dosagens de lipase pancreática por imunorreatividade demonstrando valores acima dos limites de referência. Tais autores observaram $75 \%$ de concordância entre ambos os métodos diagnósticos (ultrassonografia abdominal e Spec CPL). Entretanto, no que se refere a confiabilidade de cada uma destas metodologias, é sabido que a ultrassonografia abdominal possui cerca de $68 \%$ de sensibilidade diagnóstica, enquanto a referida dosagem enzimática apresenta cerca de $82 \%$ (Vaden et al. 2009). Os tempos de protrombina e de tromboplastina parcial ativada foram solicitados apenas para o paciente dois, devido à 
evidência ultrassonográfica de pancreatite. Destas análises, apenas o segundo dos referidos tempos de coagulação apresentou-se elevado, fato que pode se relacionar com a injúria endotelial vascular secundária à absorção do rícino para a via sistêmica, uma vez que o diagnóstico ultrassonográfico de pancreatite não foi confirmado pela dosagem de lipase pancreática.

Do ponto de vista terapêutico, não existe antídoto que anule os efeitos do rícino sobre o organismo, sugerindo-se apenas manejo protetor gastrointestinal e suporte sintomático sistêmico (Hong et al. 2011). Nos casos de ingestão poucos instantes antes da admissão hospitalar, torna-se possível a indução emética, como pela administração oral de peróxido de hidrogênio a 3\% (5 mililitros por quilograma de peso), seguido do emprego de carvão ativado (1 a 4 gramas por quilograma), para adsorção da referida substância (Albretsen et al. 2000). Nos pacientes sob debate neste trabalho, a indução do vômito não foi preconizada devido ao tempo transcorrido entre a ingestão da torta de mamona e o atendimento, porém os demais fármacos empregados (antieméticos, protetores gastrointestinais e antibiótico) permitiram a resolução completa das manifestações clínicas do paciente 1, diferentemente de seu contactante, o qual permaneceu com quadro entérico por 96 horas após a intoxicação. Segundo Botha \& Penrith (2009), a intensidade e o período de ocorrência dos sinais e sintomas podem depender diretamente da quantidade de substância ingerida, fato que eventualmente pode justificar as diferenças clínicas entre os animais durante a admissão hospitalar, bem como sua evolução clínica distinta.

\section{Conclusão}

Com base no exposto, conclui-se o contexto clínico, imaginológico e laboratorial das intoxicações por rícino em cães, especialmente no que se refere à ingestão de sua apresentação sob o adubo torta de mamona, fato que inclui a predominância das manifestações clínicas gastrointestinais, porém, com possibilidade de envolvimento do sistema circulatório, o tempo médio de hospitalização de 48 horas, a necessidade de monitorização clínica, hematológica, bioquímica e ultrassonográfica gastrointestinal, e ainda a importância da instituição precoce do manejo terapêutico, mesmo que sintomático, para que haja otimização da recuperação do paciente.

\section{Referências bibliográficas}

Albretsen J.C., Gwaltney-Brant S.M. \& Khan S.A. 2000. Evaluation of castor bean toxicosis in dogs: 98 cases. Journal of the American Animal Hospital Association 36, 229-233.

Aleixo G.S., Coelho M.C.O., Tenório A.P.M., Guimarães A.L.N., Andrade M.B. \& Cavalcanti H.B. 2010. Uso do glicosímetro portátil para determinar a concentração de glicose no sangue de cães. Ciência Animal Brasileira 11, 537-545.

Baltazar F.N., Januário E.V., Trevizan R., Berl C.A., Nogueira F.R. \& Duarte R. 2016. Alterações clínicas, hemostáticas e tempo de hospitalização em cães com pancreatite aguda: 71 casos (2013-2015). In: Anais do $37^{\circ}$ Congresso Brasileiro da Associação Nacional de Clínicos Veterinários de Pequenos Animais, Goiânia, Goiás, Brasil.

Botha C.J. \& Penrith M.-L. 2009. Potential plant poisonings in dogs and cats in southern Africa. Journal of the South African Veterinary Association 80, 63-74.

Cangemi J.M., Santos A.M. \& Claro-Neto S.A. 2010. Revolução verde da mamona. Química Nova 32, 3-8.

Fonseca N.B.S. \& Soto-Blanco B. 2014. Toxicidade da ricina presente nas sementes de mamona. Semina: Ciências Agrárias 35, 14151424.

$\mathrm{Fu}$ T., Burbage C., Tagge E.P., Brothers T., Willingham M.C. \& Frankel A.E. 1996. Ricin toxin contains three lectin sites which contribute to its in vivo toxicity. International Journal of Immunopharmacology 18, 685-692.

Hong I.-H., Kwon T.-E., Lee S.-K., Park J.-K., Ki M.-R., Park S.-I. \& Jeong K.-S. 2011. Fetal death of dogs after the ingestion of a soil conditioner. Experimental and Toxicologic Pathology 63, 113-117.

Roels S., Coopman V., Vanhaelen P. \& Cordonnier J. 2010. Lethal ricin intoxication in two adult dogs: toxicologic and histopathologic findings. Journal of Veterinary Diagnostic Investigation 22, 466-468.

Soto-Blanco B., Sinhorini I.L., Gorniak S.L. \& Schumaher-Henrique B. 2002. Ricinus communis cake poisoning in a dog. Veterinary and Human Toxicology 44, 155-156.

Tokarnia C.H. \& Döbereiner J. 1997. Imunidade cruzada pelas sementes de Abrus precatorius e 
Ricinus communis em bovinos. Pesquisa Veterinária Brasileira 17, 25-35.

Vaden S.L., Knoll J.S., Smith F.W.K. \& Tilley L.P. 2009. Blackwell's five-minute veterinary consult: laboratory tests and diagnostic procedures (Canine \& Feline). WileyBlackwell, Ames, Iowa, USA.
Recebido: 24 outubro, 2018.

Aprovado: 17 novembro, 2018.

Publicado: 26 dezembro, 2018

Licenciamento: Este artigo é publicado na modalidade Acesso Aberto sob a licença Creative Commons Atribuição 4.0 (CC-BY 4.0), a qual permite uso irrestrito, distribuição, reprodução em qualquer meio, desde que o autor e a fonte sejam devidamente creditados. 